Mem. S.A.It. Vol. 75, 282

(C) SAIt 2008

Memorie della

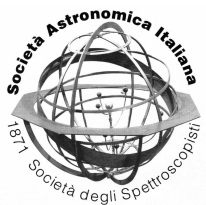

\title{
Atmospheres from very low-mass stars to extrasolar planets
}

\author{
F. Allard \& D. Homeier
}

CRAL, UMR 5574, CNRS, Université de Lyon, École Normale Supérieure de Lyon, 46 Allée d'Italie, F-69364 Lyon Cedex 07, France e-mail: fallard@ens-lyon. fr

\begin{abstract}
Within the next few years, several instruments aiming at imaging extrasolar planets will see first light. In parallel, low mass planets are being searched around red dwarfs which offer more favorable conditions, both for radial velocity detection and transit studies, than solar-type stars. We review recent advancements in modeling the stellar to substellar transition. The revised solar oxygen abundances and cloud models allow to reproduce the photometric and spectroscopic properties of this transition to a degree never achieved before, but problems remain in the important M-L transition characteristic of the $T_{\text {eff }}$ range of characterisable exoplanets.
\end{abstract}

Key words. Stars: atmospheres - M dwarfs - Brown Dwarfs - Extrasolar Planets

\section{Introduction}

Since spectroscopic observations of very low mass stars (late 80s), brown dwarfs (mid 90s), and extrasolar planets (mid 2000s) are available, one of the most important challenges in modeling their atmospheres and spectroscopic properties lies in high temperature molecular opacities and cloud formation. $\mathrm{K}$ dwarfs show the onset of formation metal hydrides (starting around $T_{\text {eff }} \sim 4500 \mathrm{~K}$ ), $\mathrm{TiO}$ and $\mathrm{CO}$ (below $T_{\text {eff }} \sim 4000 \mathrm{~K}$ ), while water vapor forms in early $\mathrm{M}$ dwarfs $\left(T_{\text {eff }} \sim 3900-2000 \mathrm{~K}\right)$, and methane, ammonia and carbon dioxide are detected in late-type brown dwarfs ( $T_{\text {eff }}$ $\sim 300-1600 \mathrm{~K})$ and in extrasolar giant planets. Cloud formation is also an important factor in the detectability of biosignatures, and for the habitability of exoplanets (Paillet et al. 2005, Kasting 2001).

Send offprint requests to: F. Allard
Extrasolar planets for which we can currently characterize their atmospheres are either those observed by transit $\left(T_{\text {eff }} \sim 1000-2000 \mathrm{~K}\right.$ depending on their radius relative to that of the central star) or by imaging (young planets of $T_{\text {eff }} \sim 500-2000 \mathrm{~K}$ depending on their mass and age). Several infrared integral field spectrographs combined with coronagraph and adaptive optic instruments are coming online before 2013 (SPHERE at the VLT, the Gemini Planet Imager at Gemini south, Project1640 at Mount Palomar, etc.). The E-ELT $41 \mathrm{~m}$ telescope in Spain due around 2020 will also be ideally suited for planet imaging.

M dwarfs are the most numerous stars, constituting $80 \%$ of the stellar budget of the Galaxy, and around 600 brown dwarfs and planets are currently known despite their faintness in the solar neighborhood vicinity. Single very low mass (VLM) stars and brown dwarfs are therefore more directly observable and 
characterizable then exoplanets. They represent, beyond their own importance, a wonderful testbed for the understanding of exoplanetary atmospheric properties together with solar system studies. Planets can even share the atmospheric composition of brown dwarfs of same $T_{\text {eff }}$ (see section 5 below).

The models developed for VLMs and brown dwarfs are therefore a unique tool, if they can explain the stellar-substellar transition, for the characterization of imaged exoplanets. In this paper, we review the ability of recently published models in reproducing constraints along the M-L-T spectral transition.

\section{Model Construction}

The modeling of the atmospheres of VLMs has evolved (as here illustrated with the development of the PHOENIX atmosphere code) with the extension of computing capacities from an analytical treatment of the transfer equation using moments of the radiation field (Allard 1990), to a line-by-line opacity sampling in spherical symmetry (Allard et al. 1994, 1997 and Hauschildt et al. 1999) and more recently to $3 \mathrm{D}$ radiation transfer (Seelmann et al.2010). In parallel to detailed radiative transfer in an assumed static environment, hydrodynamical simulations have been developed to reach a realistic representation of the granulation and its induced line shifts for the sun and sun-like stars (see e.g. the review by Freytag et al. 2012) by using a non-grey (multi-group binning of opacities) radiative transfer with a pure blackbody source function (scattering is neglected).

To illustrate the various assumptions made by constructing model atmospheres, let us begin with the description of the equations of ideal magnetohydrodynamics (MHD) adapted here for the stellar case by specifying the role of gravity, radiative transfer, and energy transport - which are themselves a special case (no resistivity) of the more general equations (see for example Landau \& Lifshitz
1960). These are written in the compact vector notation as:

$$
\begin{aligned}
\frac{\partial \rho}{\partial t}+\nabla \cdot(\rho \mathbf{v}) & =0 \\
\frac{\partial \rho \mathbf{v}}{\partial t}+\nabla \cdot\left(\rho \mathbf{v v}+\left(P+\frac{1}{2} \mathbf{B} \cdot \mathbf{B}\right) \mathbf{I}-\mathbf{B B}\right) & =\rho \mathbf{g} \\
\frac{\partial \mathbf{B}}{\partial t}+\nabla \cdot(\mathbf{v B}-\mathbf{B v}) & =0 \\
\frac{\partial \rho e_{\mathrm{t}}}{\partial t}+\nabla \cdot\left(\left(\rho e_{\mathrm{t}}+P+\frac{1}{2} \mathbf{B} \cdot \mathbf{B}\right) \mathbf{v}\right. & \\
\left.-(\mathbf{v} \cdot \mathbf{B}) \mathbf{B}+\mathbf{F}_{\mathrm{rad}}\right) & =0
\end{aligned}
$$

The vectors are noted with boldface characters, while scalars are not. For example, $P$ is the gas pressure, $\rho$ the mass density, $g$ the gravity, and $\mathbf{v}$ is the gas velocity at each point in space. $\mathbf{B}$ is the magnetic field vector, where the units were chosen such that the magnetic permeability $\mu$ is equal to one. I is the identity matrix and $\mathbf{a} \cdot \mathbf{b}=\sum_{k} a_{k} b_{k}$ the scalar product of the two vectors $\mathbf{a}$ and $\mathbf{b}$. The dyadic tensor product of two vectors $\mathbf{a}$ and $\mathbf{b}$ is the tensor $\mathbf{a b}=\mathbf{C}$ with elements $c_{m n}=a_{m} b_{n}$ and the $n$th component of the divergence of the tensor $\mathbf{C}$ is $(\nabla \cdot \mathbf{C})_{n}=\sum_{m} \partial c_{m n} / \partial x_{m}$. In this case, the total energy is given by

$\rho e_{\mathrm{t}}=\rho e_{\mathrm{i}}+\rho \frac{1}{2} \mathbf{v} \cdot \mathbf{v}+\frac{1}{2} \mathbf{B} \cdot \mathbf{B}+\rho \Phi$,

where $e_{\mathrm{i}}$ is again the internal energy per unit mass, and $\Phi$ the gravitational potential. The additional constraint for the absence of magnetic monopoles,

$\nabla \cdot \mathbf{B}=0$,

must also be fulfilled.

The first, third, and last equations in eq. 11 correspond to the mass, magnetic field, and energy conservation, while the second equation is the budget of forces acting on the gas. In the case of stellar astrophysics, gravitational acceleration is an important source term, while the radiative flux participates in the energy budget. Further assumptions are made in the numerical solution of these equations to address different astrophysical problems in very different regimes. The chromospheres correspond to a regime of high Mach numbers and strong 
magnetic fields where ionized gas has to follow the magnetic field lines, and where the radiative transfer must be solved for the case of a non-ideal gas. The photospheric convection simulations correspond to a regime where the thermal and convective turnover timescales are comparable i.e. Mach numbers are around 1 , and the non-local radiative transfer must be solved, often for an ideal gas. And the interior convection and/or dynamo simulations correspond to a regime where the thermal timescale is much larger then the turnover timescale, which in turn is much larger then the acoustic timescale. The radiative flux can be approximated by the diffusion approximation, and the magnetic field lines are dragged by the ionized gas.

Radiation hydrodynamical (RHD) simulations ignore by definition the magnetic field terms in equation 1. This is a good approximation when modeling the neutral photosphere (where most of the emitted flux emerges) of low mass, very low mass stars, and brown dwarfs - with the exception of the ultraviolet and visual spectral range of flaring stars and for the resulting emission lines. RHD simulations, especially in $2 \mathrm{D}$ and $3 \mathrm{D}$, are computationally expensive, and, when treating - if at all - radiative transfer, can currently be performed only for a restricted number of wavelengths, or wavelength bins (typically 4 to 12). In the case of solar-like photospheres, RHD simulations using $140^{2} \times 150$ grid points over 5 hours of stellar time CO5BOLD required on parallel computers (2 nodes) several CPU months (Ludwig et al. 2009). In the case of red and brown dwarf simulations, local 2D cases with a resolution of $400 \times 300$ covering 2 days of stellar time CO5BOLD (Freytag et al. 2010) required 1 month of CPU time.

The classical approach for interior and atmosphere models consists in simplifying the problem for a gain of computing efficiency, neglecting the magnetic field, convective and/or rotational motions and other multidimensional aspects of the problem, and assuming that the averaged properties of stars can be approximated by modeling their properties radially (uni-dimensionally) and statically. We also assume that the atmosphere does nei- ther create nor destroy the radiation emitted through it. Neglecting motions in modeling the photospheres of VLM stars, brown dwarfs, and planets is acceptable since the convective velocity fluctuation effects on line broadening is hidden by the strong van der Waals broadening prevailing in these atmospheres. But this is not the case of the impact of the velocity fields on the cloud formation and wind processes (see section 4 below). In this case, equation 1 reduces to the so-called hydrostatic equation and constant flux approximation for the radial or $z$ direction used in classical models:

$$
\begin{gathered}
\frac{\partial P}{\partial r}=-\rho g, \\
\frac{\partial F_{\mathrm{rad}}}{\partial r}=\frac{\partial\left(\int F_{\lambda} \mathrm{d} \lambda\right)}{\partial r}=0 .
\end{gathered}
$$

This allows computing the interior evolutive properties of stars throughout the Hertzsprung-Russell diagram, and to solve the radiative transfer in the atmosphere for a much larger number of wavelengths (line-by-line or opacity sampling) or wavelength bins (Opacity Distribution Function or ODF, K-Coefficient) compared to R(M)HD simulations. Classical model atmospheres impose therefore the independent parameter $\mathrm{F}_{\text {rad }}\left(=\sigma T_{\text {eff }}\right.$, where $\sigma$ is the Stefan-Boltzman constant) and compute $F_{\lambda}$ so that, after model convergence, the target $F_{\text {rad }}$ is reached. Other independent parameters are the surface gravity $g$ and the abundances of the elements $\epsilon_{i}$. This makes it possible to create extensive databases of synthetic spectra and photometry that provide the basis for the interpretation of stellar observations.

All the model atmospheres compared in this review are classical models in this sense, and differ mainly in the completeness and accuracy of their opacity database ()including their cloud model assumptions), and the assumed solar abundances used for the particular grid shown. They must resolve the radiative transfer for the entire spectral energy distribution (as can be seen from eq. 4) with a good enough spectral resolution to account for all cooling and heating processes.

Classical model atmospheres differ also from one another in their construction philosophy, which is linked to their period of initial 
development. The code by (Tsuii 1965, 2002, 2005), the ATLAS code by Kurucz (1973) and Castelli \& Kurucz (2004), and the MARCS code by (Gustafsson et al. 1975, 2008) have seen the punched computer cards and the need to spend all efforts in saving characters and computer time. These models pre-tabulate their opacities $\left(\sum_{i} \kappa_{i}(\lambda)=\sum_{i} n_{i} \sigma_{i}(\lambda)\right.$, where $\kappa_{i}(\lambda)$ $\left[\mathrm{cm}^{-1}\right]$ and $\sigma_{i}(\lambda)\left[\mathrm{cm}^{2}\right]$ are the opacity coefficient and cross-section at the wavelength $\lambda$, and $n_{i}\left[\mathrm{~cm}^{-3}\right]$ is the number density of species $i$ i.e. atoms, molecules or grains) to interpolate them later during the model atmosphere execution. The PHOENIX code (Allard et al. 1994, 2012) on the other hand, also to distinguish itself from its forefathers, took the approach of computing the opacities during the model execution (or on-the-fly). This involves computing the opacities for billions of atomic and molecular transitions on-the-fly, though with a selection of the most important lines. This different approach makes PHOENIX much slower then former codes, but allows to take into account more consistently important physical phenomena, such as those involving a modification of local elemental abundances along the atmospheric structure (e.g. non-LTE, photoionisation, diffusion and cloud formation).

Therefore, and especially in the PHOENIX case, the computational requirements of classical model atmospheres, even nowadays, preclude in practice modeling globally a star from its interior to its photospheric layers. Besides, an eventual classical static 1D model appears less interesting then global RHD simulations. This is becoming possible even with rotation but of course at the cost of some severe approximations at this point: the innermost core is replaced by an adapted potential function (Steffen \& Freytag 2007). These RHD simulations of main sequence stars, brown dwarfs, and planets have also to be scaled down significantly in radius to preserve the ability to resolve convective cells and timescales of important processes such as cloud formation. An alternative approach used by many authors is therefore to neglect small scale phenomena and model only larger scales, such as global circulation around the planetary surface (see for example Koskinen et al. 2007, Showman et al. 2009, Dobbs-Dixon et al. 2010). The challenge of such hydrodynamical simulations nowadays is to account for all the most important opacities, in particular scattering, in solving the radiative transfer and hydro equations while keeping the computing time for the model within reasonable limits.

\section{Molecular opacities}

While earlier work has been developed for the study of red giant stars, the pioneering work on the modeling of VLM atmospheres has been provided by Mould (1975), Allard (1990) and Kui (1991) using a band model or the Just Overlapping Line Approximation (JOLA) opacities developed by Kivel et al. (1952) and adapted for astrophysical use by Golden (1967).

More realistic model atmospheres and synthetic spectra for VLMs, brown dwarfs. and extrasolar planets using line-by-line or opacity sampling techniques have been made possible thanks to the development of accurate opacities calculated often ab initio for atmospheric layers where temperatures can reach $3000 \mathrm{~K}$. The process of improvements was especially remarkable in the case of water vapor line lists. Indeed, water vapor has seen an important evolution through the years from band model approximations to straight means based on hot flames experiments, and then to ab initio computations. Nevertheless, the atmosphere models have failed to reproduce the strength of the water bands that shape the low resolution $(R \leq 300)$ infrared spectral energy distributions (hereafter SED) of M dwarfs. At the lower temperatures of brown dwarfs, methane and ammonia rival the effect of water. The discrepancies in the model synthetic spectra were therefore believed to be due to inaccurate or incomplete molecular opacities. In particular water vapor was suspected because the discrepancies were observed at infrared wavelengths in the relative brightnesses of the flux peaks between water vapor bands.

In Fig. 1 the models are compared to the infrared spectrum of the M8e dwarf VB10. One can see that the water vapor opacity profile, which shape this part of the spectrum, 


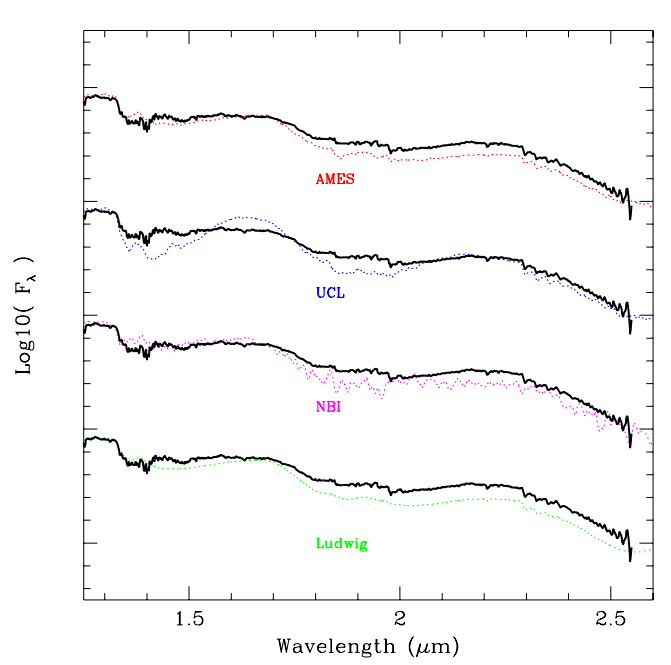

Fig. 1. Fig. 1 of the review article by Allard et al. (2012). The near-infrared SED of VB10 is compared to synthetic spectra $\left(T_{\text {eff }}=2800 \mathrm{~K}, \log g=\right.$ 5.0, $[\mathrm{M} / \mathrm{H}]=0.0, \Delta \lambda=50 \AA$ ) from diverse model grids published through the years. All models (except the NextGen/UCL case) underestimate the flux in the $K$ bandpass by 0.1 to 0.2 dex.

has strongly changed over time with the improvement of computational capacities and a better knowledge of the interaction potential surface. Only the most recent ab initio results (Partridge \& Schwenke 1997, and the BT2 line list by Barber et al. 2006) confirm the earliest hot flames laboratory experiment results by Ludwig (1971). Nevertheless, a lack of flux persist in the $K$ bandpass in the models even using the most recent BT2 opacity profile (e.g. the BT-Settl models of Allard et al. 2012). Only the UCL line list (Schryber et al. 1995, due to incompleteness, and with much of its deviations canceling out over the bandpasses) could produce seemingly correct $J-K$ colors, and could allow some success of this so-called NextGen (Hauschildt et al. 1999) model atmospheres grid in the VLM stellar regime.

In the substellar regime, the composition of brown dwarfs varies rapidly with decreasing $T_{\text {eff }}$, and the variation is responsible for the immense change in their SED across the very narrow $T_{\text {eff }}$ regime of the M-L-T spectral transition. If water vapor opacities only be- came recently reliable, this is not the case of the more complex methane molecule which is so important in brown dwarfs, and planetary atmospheres. The ExoMol Project supported by an ERC to Jonathan Tennyson (University College London) will allow important advances on these fronts in the coming years. A new ammonia line list is already available through this project (Yurchenko et al. 2011).

\section{Mixing}

Stars becomes fully convective throughout their interior and convection reaches furthest out in the optically thin regions of the photosphere in M3 and later dwarfs with $T_{\text {eff }}$ below 3200 K (Allard 1990, Chabrier \& Baraffe 2000). In most model atmospheres discussed in this review paper, the convective energy transfer is treated using the Mixing Length Theory (or MLT, see Kippenhahn \& Weigert 1994), using at best a unique fixed value of the mixing length of 1.0 (1.25 for the ATLAS9 models, 1.5 for the MARCS models, etc). However, since convection becomes efficient in M dwarfs, the precise value of the mixing length matters only for the deep atmospheric structure and as a surface boundary condition for interior models.

Ludwig et al. (2002) and Ludwig et al. (2006) have been able to compare the PHOENIX thermal structure obtained using the MLT with that of RHD simulations. They showed that the MLT could reproduce adequately (except for the overshoot region) the horizontally averaged thermal structure of the hydro simulations when using an adequate value of the mixing length parameter. This value has been estimated for M dwarfs to vary with surface gravity from $\alpha=l / H_{p}=1.8$ to 2.2 (2.5 to 3.0 for the photosphere).

The BT-Settl models use the mass and surface gravity dependent prescription of Ludwig et al. (1999) for hotter stars, together with an average (2.0) of the values derived for M dwarfs by (Ludwig et al. 2002, 2006). They use as well the micro-turbulence velocities from the radiation hydrodynamical simulations (Freytag et al. 2010), and the velocity field from RHD simulations from Ludwig et al. (2006) and Freytag et al. (2010) to calibrate the 
scale height of overshoot, which becomes important in forming thick clouds in L dwarfs but is negligible for the SED of VLMs and brown dwarfs otherwise.

Freytag et al. (2010) have indeed addressed the issue of mixing and diffusion in VLM atmospheres by 2D RHD simulations, using the PHOENIX gas opacities in a multi-group opacity scheme, and forsterite with geometric cross-sections. These simulations assume efficient nucleation, using initial monomer densities estimated from the total available density of silicon (least abundant element in the solar composition involved in forsterite). They found that gravity waves form at the internal convective-radiative boundary, and play a decisive role in cloud formation, while around $T_{\mathrm{eff}} \approx 2200 \mathrm{~K}$ the cloud layers become thick enough to initiate cloud convection, which dominates in the mixing.

\section{Atmospheric composition}

The composition of the atmospheres of stars, brown dwarfs, and planets is a function of $T_{\text {eff }}$ (radiation either due to internal heat from nuclear fusion and contraction or from irradiation by a parent star), of surface gravity to a lesser extent, and of the elemental abundances of the initial gas from which the star or stellar system is formed. Stellar model atmospheres assume scaled solar abundances for all elements relative to hydrogen. Additionally, some enrichment of $\alpha$-process elements ( $\mathrm{C}, \mathrm{O}, \mathrm{Ne}, \mathrm{Mg}, \mathrm{Si}$, $\mathrm{S}, \mathrm{Ar}, \mathrm{Ca}$, and $\mathrm{Ti}$ ) resulting from a "pollution" of the star-forming gas by the explosion of a supernova is appropriate in the case of metalpoor subdwarfs of the Galactic thick disk, halo, and globular clusters, and the stars in the high stellar density environment towards the galactic center (Gaidos et al. 2009).

\subsection{The revision of solar abundances}

Important revisions have been made to the solar abundances based on radiation hydrodynamical simulations of the solar photosphere, and to improvements in the detailed line profile analysis. Indeed, two separate groups using independent RHD and spectral synthesis codes (Asplund et al. 2009, Caffau et al. 2011) obtain an oxygen reduction of $0.11-0.19$ dex (up to $34 \%$ ) compared to the previously used abundances of Grevesse et al. (1993). Since the overall SED of late K dwarfs, M dwarfs, brown dwarfs, and exoplanets is governed by oxygen compounds ( $\mathrm{TiO}, \mathrm{VO}$ in the optical and water vapor and $\mathrm{CO}$ in the infrared), the elemental oxygen abundance is of major importance. Fig. 2] shows an example of these effects, where several models are compared to the optical to infrared SED of the M5.5, M9.5, and L0 dwarfs of the LHS 1070 system. The BT-Settl model by Allard et al. (2012) is based on the Asplund et al. (2009) solar abundance values, while DRIFT models by Helling et al. (2008b) use the Grevesse et al. (1993) solar abundances, and the MARCS model by Gustafsson et al. (2008) uses the values of Grevesse et al. (2007). Inspecting Fig. 2, one can see that the MARCS model show a systematic near-infrared flux excess, compared both to observations and the other models, which is probably caused by the much lower oxygen abundance values of Grevesse et al. (2007). The oxygen abundances sensitivity of $\mathrm{TiO}$ bands is expressed as a reduced line blanketing effect at longer wavelengths, participating in the water vapor profile changes (Allard et al. 2000).

The influence of the solar oxygen abundance can also be clearly seen in Fig. 3 which compares the Casagrande et al. (2008) $T_{\text {eff }}$ and metallicity estimates with the Baraffe et al. (1998) NextGen isochrones (assuming an age of 5 Gyrs) using model atmospheres from various authors. The oxygen abundance effects are particularly highlighted by comparing the BT-Settl model based on the Asplund et al. (2009) values with models based on earlier solar abundance values. This is the case of the AMES-Cond/Dusty and BT-NextGen models by Allard et al. $(2001,2012)$ which are based on the Grevesse et al. (1993) solar abundances. On can see that the higher oxygen abundance causes models to appear too blue by as much as $0.75 \mathrm{mag}$ compared to models based on the Asplund et al. (2009) values. The MARCS models (Gustafsson et al. 2008) based on the Grevesse et al. (2007) values show on the con- 

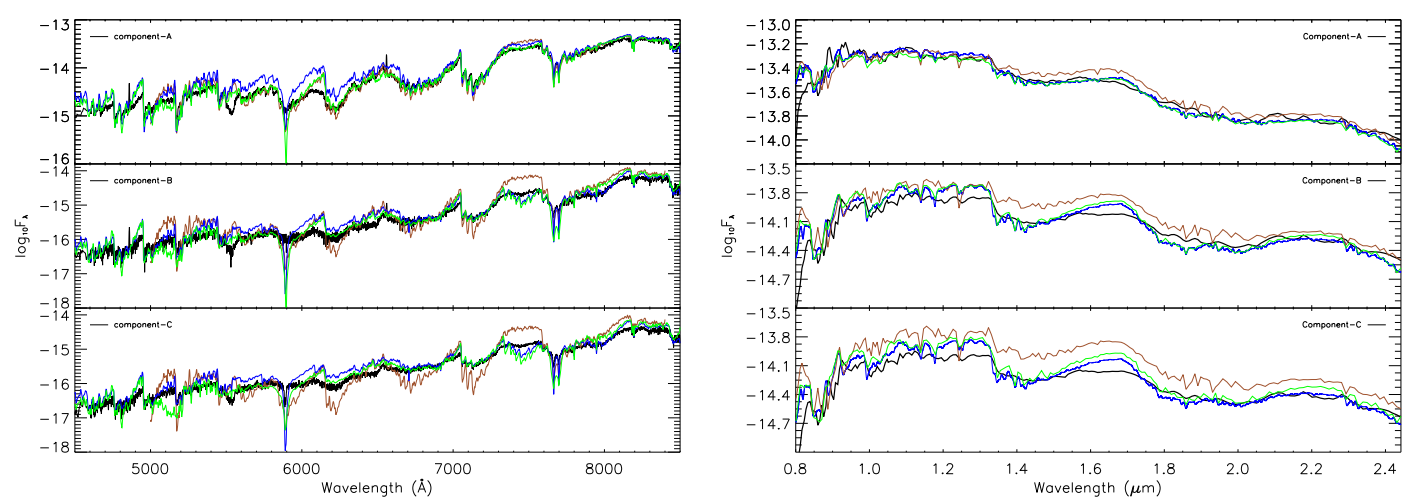

Fig. 2. Fig. 8 (left) and 9 (right) of Rajpurohit et al. (2012). In each of the three panels, the spectra of the resolved components of the LHS 1070 triple system (Leinert et al. 1994) - observed with the Faint Object Spectrograph (FOS) on the left (Leinert et al.2000) and with NICMOS on HST on the right - are compared to models of various authors. Black: observed spectra. Green: best $\chi^{2}$ fit BT-Settl AGSS model Allard et al. (2012). Blue: DRIFT model (Helling et al. 2008b). And brown: MARCS model (Gustafsson et al. 2008). The obtained parameters are given in Raipurohit et al. (2012), but do not change by more then one to two sigma from earlier estimations (Leinert et al. 2000) based on the AMES-Dusty models by Allard et al. (2001), given the grid parameter spacing $(100 \mathrm{~K})$. The $\mathrm{B}$ and $\mathrm{C}$ components are cool enough $\left(T_{\text {eff }}=2500\right.$ and $2400 \mathrm{~K}$ respectively) for their SED to be affected by dust formation.

trary a systematically increasing excess in $J-$ $K_{s}$ with decreasing $T_{\text {eff. }}$. The models are most sensitive on the solar oxygen abundances for $\mathrm{M}$ dwarfs around $3300 \mathrm{~K}$, i.e. at the onset of water vapor formation.

The NextGen model by Hauschildt et al. (1999) dates too far back and suffers from too much opacity differences (incompleteness essentially) to participate in this illustration. In fact, this plot helps to conclude that using the NextGen models caused a systematic overestimation of $T_{\text {eff }}$ for VLM stars. It is interesting to note that all models appear too red in the $\mathrm{K}$ dwarf range above $4000 \mathrm{~K}$. This may be due to an under representation of the $\mathrm{K}$ dwarfs in this diagram. The unified cloud model (hereafter UCM) by Tsuii (2002) show a completely different behavior in this diagram, sharing the colors of NextGen or even MARCS models at $4000 \mathrm{~K}$, but diverging towards the BT-Settl colors at $3500 \mathrm{~K}$ to finally cross-over to bluer colors as dust begin to form and affect the SED below $2600 \mathrm{~K}$.
The various model atmospheres have not been used as surface boundary condition to interior and evolution calculations, and simply provide the synthetic color tables interpolated on the published theoretical isochrones (Baraffe et al. 1998). Even if the atmospheres partly control the cooling and evolution of $\mathrm{M}$ dwarfs (Chabrier \& Baraffe 1997), differences introduced in the surface boundary conditions by changes in the model atmosphere composition have negligible effect.

\subsection{Metallicity}

The ground work in understanding the metallicity effects on the SED and colors of VLM stars has been established by Allard (1990) and Allard \& Hauschildt (1995), and summarized by Allard et al. (1997). The main effects of reducing the metallicity are the gradual disappearance of the double-metal molecules of importance for the overall opacities and the SED of VLM stars (TiO, VO, CO), and the increased pressure effects (atomic line widths, 

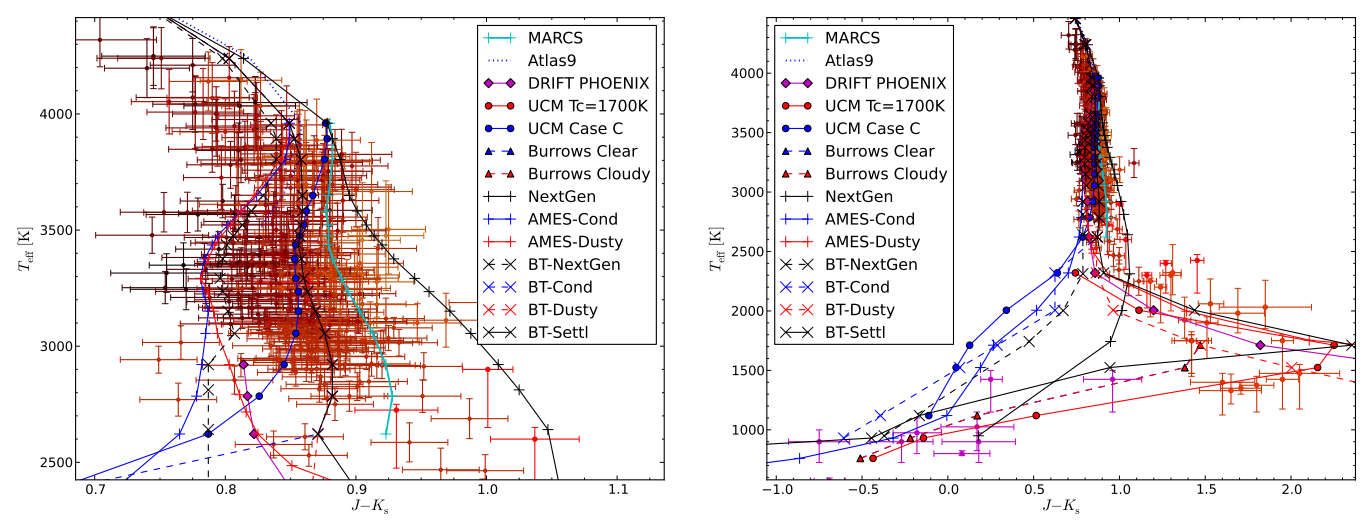

Fig. 3. Estimated $T_{\text {eff }}$ and metallicity (lighter to darker tones) for $\mathrm{M}$ dwarfs by Casagrande et al. (2008) on the left, and brown dwarfs by Golimowski et al. (2004) and Vrba et al. (2004) on the right are compared to the NextGen isochrones for 5 Gyrs Baraffe et al. (1998) using model atmospheres by various authors: MARCS by Gustafsson et al. (2008), ATLAS9 by Castelli \& Kurucz (2004), DRIFT-PHOENIX by Helling et al. (2008b), UCM by Tsuii (2002), Clear/Cloudy by Burrows et al. (2006), NextGen by Hauschildt et al. (1999), AMES-Cond/Dusty by Allard et al. (2001), and the BT models by Allard et al. (2012). The region below $2900 \mathrm{~K}$ is dominated by dust formation. The dust free models occupy the blue part of the diagram and only at best explain T dwarf colors, while the Dusty and DRIFT models explain at best L dwarfs, becoming only redder with decreasing $T_{\text {eff }}$. The BT-Settl, Cloudy and UCM $T_{\text {crit }}=1700 \mathrm{~K}$ models describe a complete transition to the red in the $\mathrm{L}$ dwarf regime before turning to the blue into the $\mathrm{T}$ dwarf regime. The Cloudy model however does not explain the reddest L dwarfs.

the strength of the hydride molecular bands including the well-known $\mathrm{H}_{2}$ collision-induced absorption (CIA) bands in the $K$ bandpass) resulting from the increased transparency of the atmosphere. This is illustrated by Fig. 5 of Allard et al. (1997), which shows how these changes operate in pushing the peak of the SED to the blue as metallicity decreases from solar to $[\mathrm{M} / \mathrm{H}]=-2.0$, and -4.0 , and the optical is becoming brighter while the CIA opacities depress the $K$-band flux.

The main difficulty in parametrizing $M$ subdwarfs using pressure indicators are due to the fact that an atmospheric pressure increase, while obtained by decreasing the metallicity, can also be provided by increasing the surface gravity, and/or reducing the effective temperature. Moreover, subdwarfs have systematically higher gravities and smaller radii than solar type M dwarfs (Baraffe et al. 1997), compensating for the increased brightness of the more transparent atmosphere at optical wavelengths. Thus, disentangling these effects can be diffi- cult to impossible using low spectral resolution or broad band colors, and detailed high resolution studies are necessary. Work is in progress using the zeta index (or $\mathrm{TiO} / \mathrm{CaH}$ flux ratio) by Lepine \& Scholz (2008) and SDSS g-r and ri colors (Bárbara Royas Ayala and Sébastien Lépine, private comm.).

In the substellar regime, the metallicity effects are similar to those enumerated above, but adapted to their cooler composition, with the difference that the SED of T dwarfs is not expected to become bluer with decreasing metallicity as for M dwarfs. This is due to the already extreme transparency of their atmosphere (due to condensation), and the resulting extreme strength and width of optical alkali spectral doublets (in particular Na ID and K I at $0.77 \mu \mathrm{m})$.

Even though some metal-poor L dwarfs have been identified by Burgasser et al. (2003, 2004, 2006) and Sivarani et al. (2009), metalpoor brown dwarfs have all chances to be $\mathrm{T}$ dwarfs by the age of the thick galactic disk 
(Population II) according to evolutionary models. For this reason, and because $\mathrm{T}$ dwarfs are more readily modeled than the dustier $\mathrm{L}$ dwarfs (see section 6 below), several studies such as those by Liu et al. (2007), Leggett et al. (2010), and Pinfield et al. (2012) have been done, despite the extreme difficulty tied to the poor spectral resolution of the observations and the fact that the SED of T dwarfs changes very slightly with the atmospheric parameters compared to those of $\mathrm{L}$ and $\mathrm{M}$ dwarfs. The submetallicity of $\mathrm{L}$ and $\mathrm{T}$ brown dwarfs is not extreme $([\mathrm{Fe} / \mathrm{H}]>-0.5)$. More metal-poor brown dwarfs would more likely be Y dwarfs.

\subsection{Disequilibrium chemistry}

Mixing has been held responsible, beyond its role in updrafting condensible gas into the cloud forming layers above, for deviations from gas phase chemical equilibrium in the atmospheres of ultracool $\mathrm{T}$ dwarfs, exoplanets, and Jupiter. These effects on their SED have been observed as an excess of carbon monoxide absorption (Noll et al. 1997, Griffith \& Yelle 1999). Similarly, ammonia has been shown to be under-abundant (Saumon et al. 2006). More recently, carbon dioxide, which under chemical equilibrium conditions is expected to form in only very small quantities in hydrogen-rich atmospheres, has been directly detected spectroscopically (Tsuji et al. 2011) and inferred from mid-infrared photometry (Burningham et al. 2011).

This is understood as the result of the fact that the formation of methane and ammonia, which are expected to dominate the carbon and nitrogen chemistries, respectively, in the low temperature limit under equilibrium conditions (Lodders \& Fegley 2006), has to compete against upmixing from the deeper and warmer atmospheric layers. If the local mixing rate is high compared with the relevant reaction rates, their high-temperature counterparts $\left(\mathrm{CO}, \mathrm{CO}_{2}\right.$, $\mathrm{N}_{2}$ ) can instead be observed in the upper atmosphere in excess of their local chemical equilibrium concentrations. To estimate the formation timescales one needs to identify the most efficient reaction path of formation and then isolate the rate-limiting, i.e. slowest, step in this path. For the conversion of nitrogen to ammonia this net reaction and limiting step is generally taken to be

$3 \mathrm{~N}_{2}+2 \mathrm{H}_{2}=\mathrm{NH}_{3}:$

$$
\mathrm{N}_{2}+\mathrm{H}_{2} \rightleftharpoons 2 \mathrm{NH}
$$

according to Lewis \& Prinn (1980), with the resulting timescale given by

$t_{\mathrm{N}_{2}}^{-1}=8.45 \times 10^{-8} e^{-8151 / T} \mathrm{~cm}^{3} \mathrm{~s}^{-1}\left[\mathrm{H}_{2}\right]$

where $T$ is the gas temperature in $\mathrm{K}$ and $\left[\mathrm{H}_{2}\right]$ the number density of molecular hydrogen. Due to the strong temperature sensitivity this makes nitrogen destruction quickly inefficient around $2000 \mathrm{~K}$, meaning that the nitrogenammonia ratio in typical brown dwarf atmospheres is fixed already in the deep convection zone.

The case for the carbon monoxide to methane conversion is more complex, where a variety of possible reaction paths and corresponding timescales have been discussed in the literature. In an extensive analysis of the reaction network Visscher et al. (2010) propose

$$
\begin{aligned}
& \mathrm{CO}+3 \mathrm{H}_{2}=\mathrm{CH}_{4}+\mathrm{H}_{2} \mathrm{O}: \\
& \mathrm{H}_{2}+\mathrm{CH}_{3} \mathrm{O} \rightleftharpoons \mathrm{CH}_{3} \mathrm{OH}+\mathrm{H}
\end{aligned}
$$

with the timescale given by

$t_{\mathrm{CO}}^{-1}=k_{\mathrm{R} 863} \frac{\left[\mathrm{H}_{2}\right]\left[\mathrm{CH}_{3} \mathrm{O}\right]}{[\mathrm{CO}]}$

and estimate a reaction constant

$k_{\mathrm{R} 863}=1.77 \times 10^{-22} T^{-3.09} e^{-3055 / T} \mathrm{~cm}^{3} \mathrm{~s}^{-1}$. The resulting timescales are at some variance with earlier estimates (Prinn \& Barshay 1977, Yung et al. 1988, Griffith \& Yelle 1999, and Schaefer $\&$ Fegley 2010), but they are generally becoming longer than atmospheric mixing timescales only in the cooler outer layers above the convection zone. This means that measurements of the $\mathrm{CO}$ abundance are a sensitive probe of overshoot and other mixing processes above the Schwarzschild boundary.

This mixing may be described by a single diffusion coefficient introduced as an additional model parameter that can be inferred from the height above which $\mathrm{CO}$ 
and $\mathrm{CH}_{4}$ should be kept fixed at their relative abundances; however this method strictly only probes mixing at this "quench level" (Saumon \& Marley 2008). The RHD simulations of Freytag et al. (2010) have allowed to model the underlying mixing processes as a function of height, although the translation from the averaged hydrodynamic velocity field to the molecular diffusion coefficient is still subject to some uncertainty: the scaling behavior of stochastic waves (in stable regions of the atmosphere, far away from the convection zones) is different from that of developed turbulence (as found - approximately - in the deep stellar convective layers or the thin cloud convection zone) or from motions in overshoot layers. Fig. 13 of Freytag et al. (2010) shows how the former may be approximated by scaling with some power of the Mach number of the flow. Spectroscopic comparison with the strength of $\mathrm{CO}$ and $\mathrm{CH}_{4}$ features, e.g. in Figs. 25, 26 of King et al. (2010) demonstrates that these latter chemical models with relatively fast rates are most consistent with observations.

\section{Cloud formation}

One of the most important challenges in modeling these atmospheres is the formation of clouds. Tsuji et al. (1996) had identified dust formation by recognizing the condensation temperatures of hot dust grains (enstatite, forsterite, corundum: $\mathrm{MgSiO}_{3}, \mathrm{Mg}_{2} \mathrm{SiO}_{4}$, and $\mathrm{Al}_{2} \mathrm{O}_{3}$ crystals) to occur in the line-forming layers $\left(\tau \approx 10^{-4}-10^{-2}\right)$ of their models. The onset of this phase transition occurs in M dwarfs below $T_{\text {eff }}=3000 \mathrm{~K}$, but the cloud layers are too sparse and optically thin to affect the SED above $T_{\text {eff }}=2600 \mathrm{~K}$. The cloud composition, according to equilibrium chemistry, is going from zirconium oxide $\left(\mathrm{ZrO}_{2}\right)$, refractory ceramics (perovskite and corundum; $\mathrm{CaTiO}_{3}$, $\mathrm{Al}_{2} \mathrm{O}_{3}$ ), silicates (e.g. forsterite; $\mathrm{Mg}_{2} \mathrm{SiO}_{4}$ ), to salts $(\mathrm{CsCl}, \mathrm{RbCl}, \mathrm{NaCl})$, and finally to ices $\left(\mathrm{H}_{2} \mathrm{O}, \mathrm{NH}_{3}, \mathrm{NH}_{4} \mathrm{SH}\right)$ as brown dwarfs cool down over time from $\mathrm{M}$ through $\mathrm{L}, \mathrm{T}$, and $\mathrm{Y}$ spectral types Allard et al. 2001, Fergley \& Lodders 2006). This crystal formation causes the weakening and vanishing of $\mathrm{TiO}$ and $\mathrm{VO}$ molecular bands (via $\mathrm{CaTiO}_{3}, \mathrm{TiO}_{2}$, and $\mathrm{VO}_{2}$ grains) from the optical spectra of late $\mathrm{M}$ and $\mathrm{L}$ dwarfs, revealing $\mathrm{CrH}$ and $\mathrm{FeH}$ bands otherwise hidden by the molecular pseudocontinuum, and the resonance doublets of alkali transitions which are only condensing onto salts in late-T dwarfs. The scattering effects of this fine dust is Rayleigh scattering which provides veiling to the optical SED, while the greenhouse effect due to the dust cloud causes their infrared colors to become extremely red compared to those of hotter dwarfs. The upper atmosphere, above the cloud layers, is depleted from condensible material and significantly cooled down by the reduced or missing pseudo-continuum opacities.

One common approach has been to explore the limiting properties of cloud formation. One limit is the case where sedimentation or gravitational settling is assumed to be fully efficient. This is the case of the Case B model of Tsuji (2002), the AMES-Cond model of Allard et al. (2001), the Clear model of Burgasser et al. (2002), and the Clear model of Burrows et al. (2006). The other limit is the case where gravitational settling is assumed inefficient and dust, often only forsterite, forms in equilibrium with the gas phase. This is the case of of the Case A model of Tsuii (2002), the AMES-Dusty models of Allard et al. (2001), the BT-Dusty models of Allard et al. (2012), the Dusty model of Burgasser et al. (2002), and the Cloudy model of Burrows et al. (2006). To these two limiting cases we can add a third case also explored by several, which is the case where condensation is not efficient and the phase transition does not take place. This is the case of the NextGen models of Hauschildt et al. (1999), of the BTNextGen models of Allard et al. (2012), and the Case B models of (Tsuii 2002, not shown).

The purpose of a cloud model is to go beyond these limiting cases and define the number density and size distribution of condensates as a function of depth in the atmosphere, and as a function of the atmospheric parameters. The discovery of dust clouds in M dwarfs and brown dwarfs has therefore triggered the development of cloud models building up on pioneering work in the context of planetary atmospheres developed by Lewis (1969), Rossow 
(1978), and Lunine et al. (1989). The Lewis model is an updraft model (considering that condensation occurs in a gas bubbles advected from deeper layers). By lack of knowledge of the velocity field and diffusion coefficient of condensates in the atmospheres of the planets of the solar system, Lewis simply assumed that the advection velocity is equal to the sedimentation velocity, thereby preserving condensible material in the condensation layers. This cloud model did not account for varying grain sizes (these naturally vary as a function of depth in the cloud layers). Rossow, on the other hand, developed characteristic timescales as a function of particle size for the main microphysical processes of importance (condensation, coagulation, coalescence, and sedimentation). The curve intersections gives an estimate of the condensate number densities and mean grain sizes. However, this model made several explicit assumptions concerning the efficiency of supersaturation, the coagulation, etc.

Helling et al. (2008a) have compared different cloud models and their impact on model atmospheres of $\mathrm{M}$ and brown dwarfs. Most cloud models define the cloud base as the evaporation layer provided by equilibrium chemistry. In the unified cloud model of Tsuji et al. $(2002,2004)$ a parametrization of the radial location of the cloud top by way of an adjustable parameter $T_{\text {crit }}$ was used. This choice permits to parametrize the cloud extension effects on the spectra of these objects without resolving the cloud model equations. In principle, this approach does not allow to reproduce the stellar-substellar transition with a unique value of $T_{\text {crit }}$ since the cloud extension depends on $T_{\text {eff. }}$. Indeed, the transparent $\mathrm{T}$ dwarf atmospheres can only exist if the forsterite cloud layers retract below the line-forming regions in those atmospheres.

Ackerman \& Marley (2001) have solved the particle diffusion problem of condensates assuming a parametrized sedimentation efficiency $f_{\text {sed }}$ (constant through the atmosphere) and a mixing assumed constant and fixed to its maximum value (maximum of the inner convection zone). Burgasser et al. (2002) and Saumon \& Marley (2008) found that their socalled Cloudy models could not produce the
M-L-T spectral transition with a single value of $f_{\text {sed. }}$. This conclusion prompted them to propose a patchy cloud model Marley et al. (2010). We have not been able to obtain these models for comparison in this paper.

Allard et al. (2003) and Allard et al. (2012) have developed PHOENIX version 15.05 using the index of refraction of 55 condensible species, and a slightly modified version of the Rossow cloud model obtained by ignoring the coalescence and coagulation, and computing the supersaturation consistently. They density and grain size distribution with depth in the atmosphere is obtained by comparing the timescales for nucleation, condensation, gravitational settling or sedimentation, and mixing derived from the Mixing Length Theory for the convective mixing in the convection zones, exponential overshoot according to Ludwig et al. (2002, 2006), and from gravity waves according to Freytag et al. (2010). The cloud model is solved layer by layer inside out (bottom's up) to account for the sequence of grain species formation as a function of cooling of the gas. Among the most important species forming in the BT-Settl model are $\mathrm{ZrO}_{2}, \mathrm{Al}_{2} \mathrm{O}_{3}, \mathrm{CaTiO}_{3}, \mathrm{Ca}_{2} \mathrm{Al}_{2} \mathrm{SiO}_{7}, \mathrm{MgAl}_{2} \mathrm{O}_{4}$, $\mathrm{Ti}_{2} \mathrm{O}_{3}, \quad \mathrm{Ti}_{4} \mathrm{O}_{7}, \quad \mathrm{Ca}_{2} \mathrm{MgSi}_{2} \mathrm{O}_{7}, \quad \mathrm{CaMgSi}_{2} \mathrm{O}_{6}$, $\mathrm{CaSiO}_{3}, \mathrm{Fe}, \mathrm{Mg}_{2} \mathrm{SiO}_{4}, \mathrm{MgSiO}_{3}, \mathrm{Ca}_{2} \mathrm{SiO}_{4}$, $\mathrm{MgTiO}_{3}, \mathrm{MgTi}_{2} \mathrm{O}_{5}, \mathrm{Al}_{2} \mathrm{Si}_{2} \mathrm{O}_{13}, \mathrm{VO}, \mathrm{V}_{2} \mathrm{O}_{3}$, and Ni. At each step, the gas phase is adjusted for the depletion caused by grain formation and sedimentation. The grain sizes (a unique maximum value per atmospheric layer) are determined by the comparison of the different timescales and thus varies with depth to reach a few times the interstellar values (used in the dusty limiting case models) at the cloud base for the effective temperatures discussed in this paper. While the BT-Settl model assumes dirty spherical grains in the timescales equations to calculate the growth and settling of the grains, it only sums the opacity contributions of each species in each layer as for an ensemble of pure spherical grains.

Helling et al. (2008b) and Witte et al. (2009) modified the PHOENIX code to compute the DRIFT-PHOENIX models, considering the nucleation of only seven of the most important solids $\left(\mathrm{TiO}_{2}, \mathrm{Al}_{2} \mathrm{O}_{3}, \mathrm{Fe}, \mathrm{SiO}_{2}, \mathrm{MgO}, \mathrm{MgSiO}_{3}\right.$, 
$\left.\mathrm{Mg}_{2} \mathrm{SiO}_{4}\right)$ made of six different elements. The cloud model is based on resolving the moment equations for the dust density accounting for nucleation on seed particles and their subsequent growth or evaporation, solving from top to bottom of the atmosphere. This model assumes dirty grains mixed according to the composition of each atmospheric layer. It uses composite optical constants resulting in absorption and scattering properties of the grains that are therefore different than those of the BT-Settl models, possibly producing more opaque clouds. However, since the opacities are dominated by atomic and molecular opacities over most of the spectral distribution in this spectral type range, the impact of those differences are difficult to identify. The largest differences between the BT-Dusty, BT-Settl and DRIFT models are the differences in the local number density, the size of dust grains, as well as their mean composition, which are the direct results of the cloud model approach. The DRIFT model includes, similarly to the BT-Settl model, mixing by convection and overshooting by assuming an exponential decrease in mass exchange frequency in the radiative zone. But it neglects the contribution of the gravity waves included in the BT-Settl model.

The models using the limiting cases of maximum dust content describe adequately (given the prevailing uncertainties) the infrared colors of L dwarfs. The cloud-free limiting case models, on the other hand, allow to reproduce to some degree the colors of $\mathrm{T}$ dwarfs. But pure equilibrium chemistry models without parametrization of the cloud extension in the atmosphere cannot reproduce the observed behaviour of the M-L-T transition, the dusty models only becoming redder and dustier with decreasing $T_{\text {eff }}$, while dust-free models miss completely the reddening due to the dust greenhouse effects in the $\mathrm{L}$ dwarf regime. Fig. 3 shows this situation compared with the effective temperatures estimates obtained by integration of the observed SED (Golimowski et al. 2004, Vrba et al 2004). One can see from Fig. 3 that the late-type $M$ and early-type L dwarfs behave as if dust is formed nearly in equilibrium with the gas phase with extremely red colors in some agreement with the AMESDusty models. The BT-Settl models (full black line) reproduce the main sequence down to the L-type brown dwarf regime, before turning to the blue in the late- $\mathrm{L}$ and $\mathrm{T}$ dwarf regime as a result of the onset of methane formation in the $K_{s}$ bandpass. The BT-Settl models succeed as good as the limiting case AMES-Dusty (full red curve), BT-Dusty (dashed red curve), and $\mathrm{UCM} T_{\text {crit }}=1700 \mathrm{~K}$ (full red with big dots curve) at explaining the reddest colors of $\mathrm{L}$ dwarfs (assuming an age of 5 Gyrs). The fact that a UCM model with $T_{\text {crit }}$ value of $1700 \mathrm{~K}$ succeeds rather well in reproducing the L-T transition suggest that the cloud extension is somewhat constant through that transition. The DRIFT models, on the other hand, (magenta with diamonds curve) reach slightly less to the red and do not extend low enough in temperature to explain the L-T transition. The M-L transition is not reproduced by any of the different models, as shown by Fig. 2 where the CIFIST and BT-Settl models begin to show a $J$-band flux excess for the B and C components. This suggests that an additional element neglected thus far is at play, such as larger maybe porous grains. Indeed, all models assume thus far spherical and non-porous grains. The choice of solar abundances and the completeness of the opacity databases used is also somewhat important. One sees in Fig. 3 (on the right) that models based on the Asplund et al. (2009) solar abundances reach to redder colors in better agreement with constraints above $2000 \mathrm{~K}$ then other models. The understanding of the M-L transition between $T_{\text {eff }}=2000$ and $2400 \mathrm{~K}$ is an extremely important regime for the study of extrasolar planets...

\section{Conclusions}

We have compared the behavior of the recently published model atmospheres from various authors across the M-L-T spectral transition from $\mathrm{M}$ dwarfs through $\mathrm{L}$ type and $\mathrm{T}$ type brown dwarfs and confronted them to constraints. If the onset of dust formation is occurring below $T_{\text {eff }}=2900 \mathrm{~K}$, the greenhouse or line blanketing effects of dust cloud formation impact strongly $\left(J-K_{s}<2.0\right)$ the near-infrared SED 
of late-M and L-type atmospheres with $1300<$ $T_{\text {eff }}<2600 \mathrm{~K}$. The BT-Settl models by Allard et al. (2012) are the only models to span the entire regime. In the $\mathrm{M}$ dwarf range, the results appear to favor the BT-Settl based on the Asplund et al. (2009) solar abundances versus MARCS and ATLAS 9 models based on other values. In the brown dwarf (and planetary) regime, on the other hand, the unified cloud model by Tsuji (2002) succeeds extremely well in reproducing the constraints, while the BTSettl models also show a plausible transition. However, no models succeed in reproducing the M-L transition between 2900 and $2000 \mathrm{~K}$. This $T_{\text {eff }}$ range is similar to that of young (directly observable by imaging) and strongly irradiated planets (Hot Jupiters).

Acknowledgements. The research leading to these results has received funding from the French "Agence Nationale de la Recherche" (ANR), the "Programme National de Physique Stellaire" (PNPS) of CNRS (INSU), and the European Research Council under the European Community's Seventh Framework Programme (FP7/2007-2013 Grant Agreement no. 247060). It was also conducted within the Lyon Institute of Origins under grant ANR-10-LABX-66.

\section{References}

Ackerman, A. S. \& Marley, M. S. 2001, ApJ, 556,872

Allard, F. 1990, PhD thesis, Ruprecht Karls Univ. Heidelberg

Allard, F., Guillot, T., Ludwig, H.-G., et al. 2003, in IAU Symposium, Vol. 211, Brown Dwarfs, ed. E. Martín, 325-+

Allard, F. \& Hauschildt, P. H. 1995, ApJ, 445, 433

Allard, F., Hauschildt, P. H., Alexander, D. R., \& Starrfield, S. 1997, ARA\&A, 35, 137

Allard, F., Hauschildt, P. H., Alexander, D. R., Tamanai, A., \& Schweitzer, A. 2001, ApJ, 556,357

Allard, F., Hauschildt, P. H., Miller, S., \& Tennyson, J. 1994, ApJ, 426, L39

Allard, F., Hauschildt, P. H., \& Schwenke, D. 2000, ApJ, 540, 1005

Allard, F., Homeier, D., \& Freytag, B. 2012, Phil. Trans. A
Asplund, M., Grevesse, N., Sauval, A. J., \& Scott, P. 2009, ARA\&A, 47, 481

Baraffe, I., Chabrier, G., Allard, F., \& Hauschildt, P. H. 1997, A\&A, 327, 1054

Baraffe, I., Chabrier, G., Allard, F., \& Hauschildt, P. H. 1998, A\&A, 337, 403

Barber, R. J., Tennyson, J., Harris, G. J., \& Tolchenov, R. N. 2006, MNRAS, 368, 1087

Burgasser, A. J. 2004, ApJ, 614, L73

Burgasser, A. J. \& Kirkpatrick, J. D. 2006, ApJ, 645, 1485

Burgasser, A. J., Kirkpatrick, J. D., Burrows, A., et al. 2003, ApJ, 592, 1186

Burgasser, A. J., Marley, M. S., Ackerman, A. S., et al. 2002, ApJ, 571, L151

Burningham, B., Leggett, S. K., Homeier, D., et al. 2011, MNRAS, 414, 3590

Burrows, A., Sudarsky, D., \& Hubeny, I. 2006, ApJ, 640, 1063

Caffau, E., Ludwig, H.-G., Steffen, M., Freytag, B., \& Bonifacio, P. 2011, Sol. Phys., 268, 255

Casagrande, L., Flynn, C., \& Bessell, M. 2008, MNRAS, 389, 585

Castelli, F. \& Kurucz, R. L. 2004, A\&A, 419, 725

Chabrier, G. \& Baraffe, I. 1997, A\&A, 327, 1039

Chabrier, G. \& Baraffe, I. 2000, ARA\&A, 38, 337

Dobbs-Dixon, I., Cumming, A., \& Lin, D. N. C. 2010, ApJ, 710, 1395

Freytag, B., Allard, F., Ludwig, H., Homeier, D., \& Steffen, M. 2010, A\&A, 513, A19+

Freytag, B., Steffen, M., Ludwig, H.-G., et al. 2012, J. Comp. Phys., 231, 919

Gaidos, E., Krot, A. N., \& Huss, G. R. 2009, ApJ, 705, L163

Golden, S. A. 1967, JQRST, 7, 225

Golimowski, D. A., Leggett, S. K., Marley, M. S., et al. 2004, AJ, 127, 3516

Grevesse, N., Asplund, M., \& Sauval, A. J. 2007, Space Sci. Rev., 130, 105

Grevesse, N., Noels, A., \& Sauval, A. J. 1993, A\&A, 271, 587

Griffith, C. A. \& Yelle, R. V. 1999, ApJ, 519, L85

Gustafsson, B., Bell, R. A., Eriksson, K., \& Nordlund, A. 1975, A\&A, 42, 407

Gustafsson, B., Edvardsson, B., Eriksson, K., 
et al. 2008, A\&A, 486, 951

Hauschildt, P. H., Allard, F., \& Baron, E. 1999, ApJ, 512, 377

Helling, C., Ackerman, A., Allard, F., et al. 2008a, MNRAS, 391, 1854

Helling, C., Dehn, M., Woitke, P., \& Hauschildt, P. H. 2008b, ApJ, 675, L105

Kasting, J. F. 2001, AGU Fall Meeting Abstracts, $\mathrm{C} 1$

King, R. R., McCaughrean, M. J., Homeier, D., et al. 2010, A\&A, 510, A99+

Kippenhahn, R. \& Weigert, A. 1994, Stellar Structure and Evolution, ed. Kippenhahn, R. \& Weigert, A.

Kivel, B., Mayer, H., \& Bethe, H. 1952, Ann. Phys., 2, 57

Koskinen, T. T., Aylward, A. D., Smith, C. G. A., \& Miller, S. 2007, ApJ, 661, 515

Kui, R. 1991, PhD thesis, National University Australia

Kurucz, R. L. 1973, PhD thesis, HARVARD UNIVERSITY.

Landau, L. D. \& Lifshitz, E. M. 1960, Electrodynamics of continuous media, ed. Landau, L. D. \& Lifshitz, E. M.

Leggett, S. K., Burningham, B., Saumon, D., et al. 2010, ApJ, 710, 1627

Leinert, C., Allard, F., Richichi, A., \& Hauschildt, P. H. 2000, A\&A, 353, 691

Leinert, C., Weitzel, N., Richichi, A., Eckart, A., \& Tacconi-Garman, L. E. 1994, A\&A, 291, L47

Lépine, S. \& Scholz, R.-D. 2008, ApJ, 681, L33

Lewis, J. S. 1969, Icarus, 10, 365

Lewis, J. S. \& Prinn, R. G. 1980, ApJ, 238, 357

Liu, M. C., Leggett, S. K., \& Chiu, K. 2007, ApJ, 660, 1507

Lodders, K. \& Fegley, Jr., B. 2006, Chemistry of Low Mass Substellar Objects, ed. Mason, J. W. (Springer Verlag), 1-+

Ludwig, C. B. 1971, Appl. Opt., 10, 1057

Ludwig, H.-G., Allard, F., \& Hauschildt, P. H. 2002, A\&A, 395, 99

Ludwig, H.-G., Allard, F., \& Hauschildt, P. H. 2006, A\&A, 459, 599

Ludwig, H.-G., Caffau, E., Steffen, M., et al. 2009, Mem. Soc. Astron. Italiana, 80, 711

Ludwig, H.-G., Freytag, B., \& Steffen, M. 1999, A\&A, 346, 111
Lunine, J. I., Hubbard, W. B., Burrows, A., Wang, Y.-P., \& Garlow, K. 1989, ApJ, 338, 314

Marley, M. S., Saumon, D., \& Goldblatt, C. 2010, ApJ, 723, L117

Mould, J. R. 1975, A\&A, 38, 283

Noll, K. S., Geballe, T. R., \& Marley, M. S. 1997, ApJ, 489, L87+

Paillet, J., Selsis, F., \& Allard, F. 2005, in Protostars and Planets V, 8341

Partridge, H. \& Schwenke, D. W. 1997, J. Comp. Phys., 106, 4618

Pinfield, D. J., Burningham, B., Lodieu, N., et al. 2012, MNRAS, 2821

Prinn, R. G. \& Barshay, S. S. 1977, Science, 198, 1031

Rajpurohit, A. S., Reyle, C., Schultheis, M., et al. 2012, å

Rossow, W. B. 1978, Icarus, 36, 1

Saumon, D. \& Marley, M. S. 2008, ApJ, 689, 1327

Saumon, D., Marley, M. S., Cushing, M. C., et al. 2006, ApJ, 647, 552

Schaefer, L. \& Fegley, B. 2010, Icarus, 208, 438

Schryber, J. H., Miller, S., \& Tennyson, J. 1995, JQSRT, 53, 373

Seelmann, A. M., Hauschildt, P. H., \& Baron, E. 2010, A\&A, 522, A102+

Showman, A. P., Fortney, J. J., Lian, Y., et al. 2009, ApJ, 699, 564

Sivarani, T., Lépine, S., Kembhavi, A. K., \& Gupchup, J. 2009, ApJ, 694, L140

Steffen, M. \& Freytag, B. 2007, Astronomische Nachrichten, 328, 1054

Tsuji, T. 1965, PASJ, 17, 152

Tsuji, T. 2002, ApJ, 575, 264

Tsuji, T., Nakajima, T., \& Yanagisawa, K. 2004, ApJ, 607, 511

Tsuji, T., Ohnaka, K., \& Aoki, W. 1996, A\&A, 305, L1

Tsuji, T., Yamamura, I., \& Sorahana, S. 2011, ApJ, 734, 73

Visscher, C., Moses, J. I., \& Saslow, S. A. 2010, Icarus, 209, 602

Vrba, F. J., Henden, A. A., Luginbuhl, C. B., et al. 2004, AJ, 127, 2948

Witte, S., Helling, C., \& Hauschildt, P. H. 2009, A\&A, 506, 1367

Yung, Y. L., Drew, W. A., Pinto, J. P., \& Friedl, 
R. R. 1988, Icarus, 73, 516

Yurchenko, S. N., Barber, R. J., \& Tennyson, J.

2011, MNRAS, 413, 1828 